\title{
Les langues polynésiennes : obstacles et atouts
}

\section{Edgar Tetahiotupa}

\section{(2) OpenEdition}

\section{Journals}

\section{Édition électronique}

URL : http://journals.openedition.org/jso/128

DOI : $10.4000 /$ jso. 128

ISSN : $1760-7256$

\section{Éditeur}

Société des océanistes

\section{Édition imprimée}

Date de publication : 1 décembre 2004

Pagination : 139-153

ISSN : 0300-953x

\section{Référence électronique}

Edgar Tetahiotupa, «Les langues polynésiennes : obstacles et atouts », Journal de la Société des

Océanistes [En ligne], 119 | Année 2004-2, mis en ligne le 01 décembre 2007, consulté le 30 avril 2019 URL : http://journals.openedition.org/jso/128 ; DOI : 10.4000/jso.128 


\section{Les langues polynésiennes : obstacles et atouts}

par

Edgar TETAHIOTUPA*

\begin{abstract}
While French Polynesia is thought to be particularly advanced when it comes to teaching native languages in the Pacific, we realize that thirty years after the first experiments took place, teaching has not evolved in primary schools. And yet, more and more actions promote Polynesian languages: written and oral literary contests; teaching those native languages in primary schools, in secondary schools and at the university; the organization of a day dedicated to Polynesian languages and so on. This article tries to note down all the obstacles in the development of Polynesian languages and to determine why the obstacles persist. It shows our particular interest to preserve and teach these languages.
\end{abstract}

KEYwORDs: polynesians' languages, bilingualism, linguistic politics, written, Ronjat's principle, immersion.
Les premières expériences officielles d'enseignement des langues polynésiennes ont débuté vers le milieu des années dix-neuf cent soixantedix et ne concernaient que le tahitien. Elles eurent lieu en primaire et portaient en elles un nouvel espoir dans la lutte contre l'échec scolaire et dans une meilleure intégration de l'enfant polynésien dans le système éducatif. L'année scolaire 1976-1977 fut choisie comme année d'expérimentation. Une commission pédagogique formée à cet effet fut chargée d'établir des rencontres de travail avec les membres de l'Académie tahitienne, afin de réfléchir à un enseignement adapté aux petites classes. Le premier bilan effectué en mars 1977 laissa apparaître une difficulté d'une importance capitale. En effet, on se rendit compte que les maîtres n'avaient ni les compétences ni l'aisance nécessaires pour enseigner la langue tahitienne. Le projet d'extension de l'expérience à une centaine de classes prévu pour la rentrée de septembre 1978 fut ainsi abandonné. Face à cette situation, il devint urgent de

* Docteur en anthropologie, edte@mail.pf 
former les enseignants à l'enseignement de la langue tahitienne. L'École normale assure cette formation depuis une trentaine d'années. Quel bilan pouvons-nous faire aujourd'hui ? Il n'est ni très élogieux, ni très encourageant. Actuellement, en 2004, et ce n'est pas une caricature, nous en sommes encore aux expérimentations de l'enseignement du tahitien en maternelle. Est-ce à dire qu'il n'y eut point d'avancées depuis 1977 ? Si ! bien sûr, mais elles furent disparates, très inégales et inconstantes.

En comparaison avec les deux autres territoires français du Pacifique, la Nouvelle-Calédonie et Wallis et Futuna, on peut considérer la Polynésie française d'il y a trente ans comme étant en avance en matière de politique linguistique et d'enseignement des langues autochtones, pour preuve, la création de l'Académie tahitienne en 1974. Les premières formations d'élèvesinstituteurs débutent à l'École normale dans la première moitié des années dix-neuf cent soixante-dix. La langue tahitienne devient langue officielle en 1980, au même titre que la langue française. L'extension de la loi Deixonne', en 1981, permet la mise en place de l'enseignement des langues et dialectes locaux et le tahitien est admis au baccalauréat. Les premiers textes rendant obligatoire l'enseignement des langues polynésiennes en maternelle et en cycle élémentaire apparaissent en 1982. L'année 2000 voit la création de l'Académie marquisienne. En Nouvelle-Calédonie, durant les années dix-neuf cent quatre-vingts, période d'instabilité politique, il fut mis en place, dans de nombreuses régions, des écoles populaires kanak. Elles sont nées d'une décision politique, à la suite du congrès fondateur du Front national de libération kanak socialiste. Mais déjà, en 1978, une section langues vernaculaires avait été créée au Centre territorial de recherche et de documentation pédagogiques (CTRDP). Elle était chargée de mettre en place des enseignements dans différentes langues kanak et, à cette fin, d'élaborer quelques documents pédagogiques. Mais, il faudra attendre l'accord de Nouméa en 1998 pour voir s'accélérer le processus de reconnaissance des langues kanak comme des langues d'enseignement et de culture. Ainsi se rend-on compte, dans les dispositions prises en métropole par le ministère de la Jeunesse, de l'Éducation et de la Recherche, que la Nouvelle-Calédonie a pris une longueur d'avance sur la Polynésie française. En effet, sur la liste des langues régionales concernées figurent les langues mélanésiennes (ajië, drehu, nengone, paicî) et le tahitien ${ }^{2}$ (et non les langues polynésiennes). Les îles Wallis-etFutuna quant à elles n'ont pas obtenu les mêmes avancées. La demande de création d'une Académie wallisienne et futunienne, proposée en 1995 par le Conseil coutumier et l'Assemblée territoriale, n'a pas obtenu satisfaction. Paris l'a jugée contraire aux textes légaux (Moyse-Faurie, 2000 : 99). Et comme un malheur n'arrive jamais seul, le Vice-rectorat s'est opposé à toute introduction des langues vernaculaires à l'école. Malgré cela, des classes pilotes en première année de maternelle ont commencé à fonctionner à Wallis et, récemment, à Futuna (en 1999). Dans les collèges et le lycée, la langue wallisienne est dispensée depuis plus d'une dizaine d'années; la langue futunienne, un peu plus tard.

En métropole, les mesures en faveur de l'enseignement des langues régionales ${ }^{3}$ ont été prises vers la moitié des années dix-neuf cent soixantedix. En Corse, c'est en 1974 que fut introduite la langue à l'école pour une heure hebdomadaire d'enseignement facultatif. Mais, c'est à partir de 1982 que se réalisent les innovations sur les langues régionales. Cette année-là est voté le premier statut particulier de l'île. Une circulaire prévoit un enseignement de la maternelle à l'université. Il peut occuper une à trois heures par semaine dans le premier degré. Une option langue régionale fut introduite aux concours des Écoles normales. Dans le second degré, l'enseignement du corse passe d'une heure à trois. Il obtient le statut de langue vivante 2 ou langue vivante 3 . Il est prévu au baccalauréat et à certains examens. En Alsace, l'allemand fut réintroduit à l'école en 1976. Mais, c'est dans les années 1970 qu'a lieu une (nouvelle) prise de conscience. En effet, l'alsacien représente non seulement un

1. Loi que le député socialiste Deixonne réussit à faire voter en 1951 et qui, dans son article III, « marque l'entrée officielle des langues régionales dans l'enseignement: "Tout instituteur qui en fera la demande pourra être autorisé à consacrer, chaque semaine, une heure d'activités dirigées à l'enseignement de notions élémentaires de lecture et d'écriture du parler local et à l'étude de morceaux choisis de la littérature correspondante. Cet enseignement est facultatif pour les élèves.” (Hagège, 1996a : 130).

2. Cela étant, les dispositions statutaires de la Polynésie française permettent l'enseignement des autres langues polynésiennes. Mais, une fois de plus, on ne peut s'empêcher de relever le caractère "dominateur" accordé au tahitien. Cette situation, faut-il le souligner, compromet grandement les chances de reconnaissance des autres langues polynésiennes par l'Union européenne. En conséquence, elles ont très peu de chance de bénéficier d'éventuels financements de sa part... en admettant, a fortiori, que le tahitien ainsi que les langues mélanésiennes, considérés comme langues régionales, puissent bénéficier de financements de l'Union européenne.

3. À titre de comparaison, nous prendrons les langues corse et alsacienne. 
élément capital du patrimoine, mais aussi un atout, facilitant l'apprentissage de l'allemand et devenant un élément important dans la communication et l'échange avec ses pays voisins. L'alsacien et l'allemand standard sont, dit-on, deux expressions d'une même langue. La première expression est régionale et orale, non uniformisée (francique et alémanique), la seconde est la langue officielle de référence à l'intérieur du domaine germanophone. La pratique du dialecte alsacien est donc fortement encouragée par une éducation bilingue français/alsacien, même si, dit-on, les enfants ne parlent plus guère ce dialecte. En 1982, une circulaire ministérielle institue l'enseignement en option des «langues et cultures régionales $»$.

Cette première comparaison laisse apparaître des avancées indéniables dans la politique linguistique et culturelle de la Polynésie française sur la Nouvelle-Calédonie ${ }^{4}$ et Wallis-et-Futuna et semble suivre la même évolution que la Corse et l'Alsace, mis à part que le tahitien fut la seule langue à obtenir le statut de langue officielle et ce, jusqu'en $1996^{5}$, et qu'actuellement la Polynésie française dispose de deux académies ${ }^{6}$. L'accord de Nouméa (1998) en prévoit une pour la Nouvelle-Calédonie, alors qu'elle a été refusée pour Wallis-et-Futuna. Comment donc, avec de telles avancées en matière de politique linguistique, la Polynésie française en est encore, quasiment, trente ans après les premières expériences d'enseignement du tahitien en maternelle, au même stade? La raison essentielle, que nous verrons plus loin, peut se définir par la conviction, encore fortement présente, que l'apprentissage des langues autochtones nuit considérablement à l'apprentissage du français, considéré comme langue de promotion et d'ouverture. Comment cette conviction a-t-elle pu émerger et perdurer jusqu'à présent? Elle a commencé avec ce que l'on a appelé « l'interdiction de parler les langues régionales » dans les écoles. Elle s'est accompagnée d'une certaine forme d'identification à la culture française au travers des contenus des programmes scolaires, identiques, à quelques adaptations près, à ceux de la métropole.

\section{La France et sa politique de francisation}

Qu'est-ce qui a motivé les autorités de cette époque à favoriser la langue française au détriment des langues régionales qui furent interdites dans les écoles et à punir ceux qui en faisaient usage? Dans son livre Le français, histoire d'un combat, Claude Hagège, reprend en leitmotiv le combat incessant du français à travers son histoire. Le linguiste Louis-Jean Calvet parle de politique linguistique coloniale de la France et précise que le français «n'est pas une langue de toute éternité ou de droit divin, il l'est devenu historiquement, à partir du dialecte francien et au cours d'un processus qui n'a rien de linguistique » (1974/1979: 44). Nous ne nous attarderons pas sur la politique de francisation en France métropolitaine 7 , si ce n'est pour analyser celle-ci à partir de Jules Ferry vers les années 1880, au moment où on entrait dans l'uniformisation nationale des programmes, au nom du principe d'égalité des chances. À cette époque, l'usage du français était imposé :

« comme unique support du savoir. L'enseignement primaire fut ainsi profondément remanié [...]. Mais surtout, pour ce qui nous intéresse ici, cet enseignement, du fait même de son inspiration républicaine, n'octroyait aucune place aux langues régionales » (Hagège, 1996a : 126).

Il en résulta une interdiction des langues régionales avec les conséquences que l'on connait. Mais se demande Bernard Poignant :

«Pourquoi ces hommes qui sont attachés aux valeurs démocratiques mènent-ils cette politique qui s'oppose aux réalités régionales? Ils ne sont sans doute pas hostiles aux langues et aux cultures régionales. Leur but n'est prioritairement pas leur élimination, mais la diffusion, avec la langue française, l'obligation scolaire, l'alphabétisation et l'éducation civique et morale, des valeurs contenues dans la Déclaration des droits de l'homme de 1789 qui est la matrice du nouveau régime. Diffuser ces valeurs auprès de tous les petits Français, c'était leur permettre d'échapper à la tutelle des anti-Républicains que l'on trouvait encore chez des notables provinciaux, parmi lesquels la hiérarchie de l'Église catholique, des membres de l'aristocratie, une partie de la bourgeoisie intellectuelle. Ainsi assiste-t-on à une sorte de défausse : le sacrifice des

4. Sauf qu'actuellement, ce territoire est en train de prendre de l'avance. En effet, une collaboration entre l'IUFM de Nouvelle-Calédonie en partenariat avec une équipe d'enseignants-chercheurs de l'université de Nouvelle-Calédonie a permis d'élaborer un projet de formation des enseignants du primaire sur les langues kanak.

5. Dans le statut de Polynésie française de 1996, la langue tahitienne perd son caractère officiel. Il faut sans doute remonter à 1992 pour connaître l'origine de ce changement avec la réforme de la Constitution qui précise que « la langue de la République est le français ».

6. Notons à cet égard que « l'Académie de la langue basque est reconnue en Espagne [...] et dans la Communauté autonome basque [...]. En France, c'est un établissement reconnu d'utilité public [...]» (Poignant, 1998).

7. Il existe une littérature conséquente qui aborde ce sujet, nous renvoyons le lecteur aux ouvrages qui le traitent et parmi ceux-ci : celui de Claude Hagège (1996a) et celui de Louis-Jean Calvet (1974/1979)... 
langues régionales, susceptibles de favoriser conservatismes et réflexes archaïsants, contre une modernisation résolue par l'unification et l'accès au savoir confiés à la langue unitaire : le français. C'est qu'à ce moment de l'histoire, le français s'estime être porteur d'une mission civilisatrice, culturelle, politique, qui ne se limite pas aux territoires englobés par l'État, mais qui s'étend au monde. Colonisation extérieure et politique intérieure se répondent, justifiées par cette vision sacrée que nous résumerons comme la prétention à l'universalité. » (2000 : 79-80)

En Polynésie française, peu d'ouvrages traitent de l'histoire de l'enseignement de la langue tahitienne. Aussi, cette partie sera plus longue. Arrivée en mars 1797, la mission protestante de la London Missionary Society se servira de la langue tahitienne pour sa mission évangélique. Elle la codifie et procède à son enseignement.

«Au cours de l'année 1805, le révérend Davies avait composé un alphabet tahitien, un vocabulaire tahitien-anglais et fixé quelques éléments de grammaire. Dès que le "roi 8 "' Pomare II eut appris l'usage de l'alphabet, il manifesta le désir d'apprendre à lire et à écrire. Il fut le premier, et probablement le meilleur, des élèves des missionnaires. Très vite, le roi sut lire et écrire couramment. L'écriture était même son occupation principale et sa meilleure récréation. » (Malé, $1974: 6$ )

Cette exemple fera école. Le missionnaire William Ellis raconte que:

« les adultes apprenaient à lire généralement plus volontiers que les enfants et plus facilement que ne l'auraient fait des personnes du même âge en Angleterre. » (Malé, 1974 : 11)

Mais cet apprentissage se réduisait à la répétition continue de mots à épeler. Connaissant l'extraordinaire facilité de mémorisation des Polynésiens, il n'est pas étonnant de constater que :

« beaucoup d'élèves savaient par cœur le livre sans être capables d'en lire une seule ligne. » (Malé, 1974 : 11)

L'anthropologue Jean-François Baré explique ainsi cette irrésistible envie de mémoriser :

«Dès 1818 , on réalise que le texte des Évangiles ou même des manuels de lecture est traité comme une nouvelle version des textes oraux des rituels, dont la mémorisation devait être impeccablement fidèle sous peine d'inefficacité. » (1985:258)
Une école fut ouverte en 1810 par le missionnaire Davies. Dès 1829, note Jacques-Antoine Morenhout, son assiduité permit aux autochtones de lire, d'écrire... Grâce au matériel d'imprimerie apporté par le missionnaire William Ellis, le roi Pomare eut le privilège de composer, le 30 juin 1817 , la première page d'un livre de lecture en tahitien dont il tira les premiers exemplaires. Des abécédaires furent imprimés par la suite en 1818, ainsi que des livres pour débutants. Dans ce travail de codification de la langue, de traduction des Saintes écritures, il s'agissait pour les missionnaires anglais de continuer leur œuvre de conversion, de christianisation, sans oublier les autres disciplines. Cette entreprise, qui marquait l'œuvre de la mission anglaise, sera contrée par l'Église catholique romaine. En effet, celle-ci voyant d'un mauvais œil l'importance grandissante que prenaient la présence anglaise et la religion protestante dans le Pacifique, chargea " par un décret du pontife Grégoire XVI du 2 juin 1833, la jeune Congrégation des Pères du SacréCour, dite de Picpus (fondée en 1825), [...] de la conversion au catholicisme de toute la Polynésie » (Malé, $1974: 13$ ).

Le protectorat fut signé en 1842 et, jusqu'en 1847, la France imposa militairement son autorité. Cette situation profita à la mission catholique. Mais celle-ci avait déjà, en 1839, les faveurs des autorités administratives qui lui accordèrent le libre exercice de sa religion. La mission, ainsi renforcée, ouvrit une école. En 1844, ce fut l'arrivée de quatre sœurs de Saint-Joseph de Cluny... Dans toute cette agitation, la population attachée dans sa grande majorité aux missionnaires de Londres voyait dans le catholicisme la religion de la France. La mauvaise volonté, affichée par les Polynésiens pour se rendre dans les écoles catholiques, incita le gouvernement du protectorat, désireux de développer l'usage du français, à employer la manière forte.

«Dans le premier enivrement de la situation nouvelle, n'a-t-on pas été avec une rare audace, jusqu'à vouloir contraindre les jeunes O'Tahitiens à abandonner leurs écoles pour celles des prêtres du Sacré-Cœur ; et quand les parents ont résisté, prenant leurs enfants par la main, ils se sont enfuis dans les bois, c'est en les menaçant qu'on leur a ordonné de les ramener. $»^{9}$

Alors que l'enseignement de la langue tahitienne était maintenu par une révision des lois de

8. Voici comment Jean-François Baré décrit la projection par les Européens de la notion de "roi" à Tahiti : « Wallis et son équipage confère à Purea le titre de "reine"; Cook voit dans Tahiti deux kingdoms, ainsi que Bligh ; Morrisson désigne "seigneurs du manoir" la catégorie intermédiaire des sous-chefs tribaux ; le futur Pomare II sera pour les gens de la London Missionary Society, "the king" ; Ellis verra des "héraults" et des "bardes" dans les messagers et les orateurs ; le protectorat français lui-même prolongera à sa manière cette conception » (1985:173).

9. « La patrie du 21 avril 1843 », in Lutteroth, cité par Malé (1974: 15). 
1845 et 1848 , l'arrêté du 7 novembre 185710 interdit aux élèves (obligation scolaire jusqu'à quatorze ans) l'usage de toute langue, autre que la langue française, même dans les jeux. Cela ne concernait que les écoles de Papeete, non celles des districts de Tahiti. Mais une ordonnance du 30 octobre 1862 rétablit bientôt la langue tahitienne avec pour objectif de faire échec à une politique de catholicisation forcée « de tous les indigènes [...] par voie administrative et bureaucratique » (Malé, 1974. : 16). Dans son article premier, l'ordonnance stipule ceci :

« l'enseignement de la langue française est obligatoire dans les écoles de districts des États du Protectorat, au même titre que celui de la langue tahitienne. " (Malé, 1974 : 16)

La mission protestante vécut des moments difficiles, rendus pénibles par l'attitude hostile des autorités françaises que venait aggraver l'affaire Pritchard. L'œuvre sera abandonnée et les écoles fermeront progressivement leurs portes :

«En 1860, seules deux Écoles françaises libres des missionnaires anglais étaient reconnues : celle de $\mathrm{M}$. Simpson, à Moorea (Académie des Mers du Sud), et celle du révérend Howe, qui en plus de l'imprimerie, dirigeait l'École normale des teachers indigènes, aidé de sa femme. » (Malé, 1974 : 17)

Les parents, ne voulant pas que leurs enfants apprennent le catéchisme romain, firent part de leurs doléances auprès de la reine Pomare IV et auprès du représentant du gouvernement central, demandant la venue de deux pasteurs protestants. Ce projet, soumis à l'Assemblée législative, fut adopté. Suivra alors une pétition, datée du 15 mai 1860, qui sera adressée à l'empereur Napoléon III :

« Nous prions notre Empereur Napoléon III, de bien vouloir choisir parmi nos coreligionnaires de France, deux missionnaires protestants français. Nous vous adressons cette pétition avec d'autant plus de confiance qu'elle a été favorablement accueillie par Monsieur le gouverneur du Bouzet... Ce sont les ministres protestants qui nous ont retiré de notre état de barbarie. Notre religion est bonne, elle nous a inspiré de l'amour envers la France qui nous protège. Nous désirons ardemment que nos enfants appren- nent la langue française ; mais nous ne voulons pas que lorsqu'ils apprennent le français, ce ne soit que dans le but de changer de religion. » (Malé, 1974: 18) ${ }^{11}$

Trois ans plus tard, leur vœu fut exaucé. Thomas Arbousset débarqua le 13 février 1863 et le pasteur Atger le 10 juillet 1864. Le 29 juin 1880, le roi Pomare v donne ses États à la France. La même année, le gouvernement de la colonie décide de laïciser l'enseignement primaire anticipant ainsi sur la métropole, où l'instruction sera rendue gratuite en 1881, obligatoire et laïque en 1882 au bénéfice des enfants de six à treize ans. En 1932, le décret du 11 décembre considérera le tahitien comme langue étrangère dans les Établissements français d'Océanie.

Un article, paru en avril $1939^{12}$, pouvait laisser croire à travers les premières lignes à une attitude plus conciliante, moins intransigeante des autorités administratives de l'époque à l'égard de la langue tahitienne. Mais, en réalité, à travers une dialectique subtile et raisonnée, il s'agissait de démonter pas à pas toute l'argumentation mise en avant pour l'enseignement de la langue locale, afin de démontrer ses tares et donc la nécessité évidente du français comme langue d'enseignement, que viendra conforter un autre article paru trois mois plus tard dans le numéro deux du même bulletin. La démarche est sans équivoque et le constat sans complaisance :

« Il est vain de gémir sur la dislocation d'une société tuée par le contact de civilisations plus évoluées. » (Bulletin pédagogique... 2, 1939)

La nécessaire recherche d'une nouvelle organisation sociale devient primordiale. Il est écrit que le tahitien :

« ne suffit pas à l'acquisition et à la compréhension [...], la mentalité était nettement restée au stade prélogique [...], la langue qui servait à la transmission de ces récits n'a pas la précision, la netteté, la clarté, la concision nécessaire ${ }^{13}[. .$.$] , il est je crois, naïf de prétendre la$ sauver en lui conservant une langue qui servait à l'époque des clans et de la cueillette [...]. Le français est le véhicule naturel d'une science dont la logique et l'abstraction ne peuvent vraiment s'encadrer parfaitement que dans une langue qui s'est formée en partie sous l'influence et pour l'expression de cette science. » (Bulletin pédagogique... 2, 1939 : 95-96)

10. Bulletin officiel des Établissements français d'Océanie 10 et 11 (octobre et novembre 1857), titre $1^{\mathrm{er}}$, article 27.

11. Il précise dans son mémoire que, vers la fin des années soixante, les élèves protestants qui voulaient suivre leur scolarité chez les Sœurs devaient embrasser la religion catholique.

12. Bulletin pédagogique et administratif des instituteurs et institutrices des Établissements français de l'Océanie 1 (avril 1939).

13. On trouve des propos identiques chez le père Patrick O’Reilly : " Comme je dis devant Maurice Jay : "Le tahitien n'est pas une langue de culture". Ce qui est une évidence. Il me répond : "Non seulement, elle n'est pas une langue de culture, mais elle empêche la culture. Une langue sert de support à la pensée, lui donne sa clarté et sa précision, etc. Or les enfants qui ne parlent que le tahitien ne possèdent qu'un moyen d'expression sans charpente, sans rigueur, l'abstraction nécessite un certain vocabulaire. Leur pensée reste nuageuse et vague. Elle n'a comme véhicule que des termes flous...”» (1982: 227). 
Ces deux articles montrent un désir manifeste d'expliquer, de justifier l'enseignement du français. Une question se pose pourtant, la pratique de la langue tahitienne a-t-elle pu se faire malgré la promulgation du décret de 1932? Au plan local, un article, paru dans un journal de Tahiti en août 1939, met en avant le nécessaire usage de la langue tahitienne dans l'instruction de l'enfant.

« L'enseignement devrait s'attacher dans les petites classes à l'étude du tahitien; plus tard seulement on apprendrait le français. C'est là une vieille doctrine des missions évangéliques. Elles ont été critiquées ; aujourd'hui elles font figure de précurseurs. » (ReyLescure, $1951:$ 304-305)

L'auteur de cet article cite l'exemple de gouverneurs de certains territoires (Indochine en 1924, Madagascar en 1929...) qui ont pris des arrêtés en faveur de l'utilisation de la langue vernaculaire dans les classes élémentaires. Par ailleurs, dans le Pacifique, en Nouvelle-Zélande par exemple, l'apprentissage de la lecture dans le premier degré est fait dans la langue vernaculaire, celui de l'anglais se faisant progressivement vers le quatrième ou cinquième degré ${ }^{14} \ldots$

« les résultats semblent également indiquer que les Polynésiens qui ont appris leur propre langue et étudié dans leur langue maternelle pendant les premières années, et n'ont abordé l'anglais que plus tard, ont obtenu un plus grand nombre de succès à l'école de médecine de Suva et en Nouvelle-Zélande. $»^{15}$ (Platten, 1951 : 296)

Cet article, paru en 1951, fait remonter la scolarisation de ces étudiants à une période antérieure à 1939, réfutant ainsi les arguments parus dans l'article du Bulletin pédagogique...

\section{Leur reconnaissance et leur introduction à l'école}

En juin 1965, lors de l'inauguration du musée Paul Gauguin à Papeari, le gouverneur Sicurani évoqua dans son discours la double culture caractérisant la Polynésie française. Un an plus tard, eurent lieu, dans l'enceinte des écoles protestantes et catholiques et ce, jusqu'en 1969, des cours de tahitien non encore inscrits au pro- gramme de l'enseignement. Dans le public, une timide réintroduction fut menée à travers des classes dites 'd'expérimentation' de 1975 à 1980, année de la reconnaissance officielle de la langue tahitienne. En 1974 naît l'Académie tahitienne, dont les statuts paraîtront au Journal officiel de la Polynésie française le 31 janvier 1975. Il faudra attendre 1985 pour que paraisse la première grammaire tahitienne officielle.

« Cette grammaire a beaucoup bénéficié des textes des missionnaires et des linguistes, qui avaient précédemment tenté de normaliser l'écriture, le vocabulaire et la syntaxe d'une langue qui s'appauvrissait au fil des années. Elle reste d'ailleurs très empreinte des écrits des évangélistes, et fait de nombreux renvois à la Bible de Nott-Howe. » (Saura, 1986 : 101)

En 1978, paraîtront les premiers fascicules ${ }^{16}$ d'apprentissage de la langue tahitienne, destinés aux enfants de l'école primaire. Quelques années plus tard, Duro Raapoto ${ }^{17}$ intégrera le service de l'Éducation. Deux autres fascicules ${ }^{18}$ sortiront : l'un pour les élèves de cours moyen, l'autre pour les maîtres. Les premiers fascicules diffèrent des suivants par la graphie, l'Académie en a adopté une, utilisée par la plupart des États voisins du Pacifique. Le linguiste Duro Raapoto trouve dans la graphie de l'Académie tahitienne une utilisation abusive de signes et en propose une plus simple. À cette confrontation, aucune issue ne semble se dessiner, chacun défendant sa vérité bec et ongles. Il s'agit bien ici de confrontation pouvant aller jusqu'à la discorde. Mais paradoxalement, le ministre de l'Éducation Nicolas Sanquer affirme, dans un article paru dans le Veà porotetani $^{19}$ (journal protestant), que la référence en matière de graphie dans l'enseignement est l'Académie tahitienne, mais en même temps, il demande à Duro Raapoto, conseiller pédagogique, de revoir le programme du secondaire avec tous les professeurs de langue $m \bar{a} \bar{c}^{\prime}$ h $i$.

$\mathrm{Si}$, comme nous l'avons vu en métropole, le droit à la reconnaissance des langues régionales fut (et demeure) un combat incessant, en Polynésie française comment cela se passa-t-il ? François Ravault, dans son article Le français dans une société pluri-culturelle rappelle que la bourgeoisie demie ${ }^{20}$, notamment les jeunes, se passionna pour la langue tahitienne, vers les années

14. C'est-à-dire des classes de CMI et CM2.

15. Selon une enquête du Survey of the Health of the Mori Language $2001,83 \%$ des adultes affirment être satisfaits ou très satisfaits des résultats obtenus en langue maorie avec les écoles d'immersion Khanga reo (nid de la langue).

16. Service de l'Éducation et Académie tahitienne, 1978, T'u puta reo tahiti, fascicule de l'élève, $1^{\mathrm{er}}$ et $2^{\mathrm{e}}$, avec un fascicule du maître, Polynésie française.

17. Duro Raapoto ou Turo Raapoto sont une seule et même personne.

18. Turo Raapoto, sd., Puta haapiiraa reo mòhi faito CM, Haapiiraaa reo na te mau òrometua haapii, service de l'Éducation.

19. Du n ${ }^{\circ} 9$, paru en novembre 1996

20. C'est-à-dire : métis, compris surtout comme ayant au moins un ascendant blanc. 
dix-neuf cent soixante-dix, tandis que les Polynésiens s'intéressèrent de plus en plus à la langue française. Parmi ces jeunes de la bourgeoisie demie, certains apprirent le tahitien à l'INALCO, à Paris. Ils ont contesté :

« le statut privilégié accordé officiellement au français [...] [Mais] il n'en a pas toujours été ainsi. Pendant longtemps en effet, exception faite de certains milieux proches des Églises, les demis ont adhéré, sans restriction apparente, à une politique de francisation qui constituait pour ses promoteurs, en Polynésie comme ailleurs, le meilleur outil de l'intégration des populations d'Outre-mer à la métropole. » (Ravault, 1982 : 98)

Par ailleurs, le discours tenu aussi bien par les parents d'élèves de l'école publique que par les enseignants, préconisait la maîtrise parfaite du français, langue véhiculaire et internationale. Mais il fallait concilier avec cela, l'impératif culturel. Ils ont donc pensé au bilinguisme, qui aurait eu pour effet de garder l'enfant dans son milieu culturel :

« de valoriser son patrimoine linguistique, de permettre une meilleure structuration de sa personnalité et peut-être un meilleur apprentissage du français par une meilleure connaissance de sa langue maternelle. » (CTAL, 1973, cité par Ravault, 1982 : 99)

Nous avons parlé de la bourgeoisie demie, des jeunes qui se sont passionnés pour la langue tahitienne. Il faudrait remonter une génération pour comprendre la naissance de cette ferveur. Contrairement aux Polynésiens, les demis, selon François Ravault, avaient une position ambiguë à l'égard du français, qui était pour eux une langue d'intégration. Cela étant, ils utilisaient aussi le tahitien dans leur relation avec les Polynésiens pour discuter affaire : métayage ou transaction commerciale. Il explique de manière judicieuse comment une langue, en l'occurrence le tahitien, considérée comme moins prestigieuse que la langue française par les demis, arrive à accéder à un statut supérieur, reconnu et revendiqué en tant que tel par ceux-ci.

« Ils ne découvrent véritablement le tahitien en tant que langue de culture qu'à partir du moment où les Polynésiens, qui ne s'étaient pas manifestés politiquement depuis la pacification des îles Sous-le-Vent à la fin $\mathrm{du} \mathrm{XIX} \mathrm{X}^{\mathrm{e}}$ siècle, donnent un contenu nouveau à la vieille revendication autonomiste. Les colons et les demis en effet, qui ont le quasi-monopole de l'expression politique pendant la première moitié $\mathrm{du} \mathrm{xx}^{\mathrm{e}}$ siècle, ne cessent de dénoncer l'aveuglement et l'autoritarisme du gouvernement central pour réclamer ce qu'on pourrait appeler une autonomie de gestion qui leur permettrait en fait de conserver et d'accentuer les privilèges coloniaux. Ceci étant, le problème des relations avec la métropole se pose en termes nouveaux à partir du moment où un leader charismatique, porté par le peuple polynésien, revendique une autonomie, voire une indépendance lors du référendum de 1958, qui s'accompagne de l'abolition de certains privilèges coloniaux, en matière fiscale et foncière notamment. La tactique de la bourgeoisie demie, qu'elle fasse partie de l'entourage de Pouvanaa ou qu'elle s'oppose ouvertement à lui, va dès lors consister, une fois le leader éliminé, à canaliser à son seul profit ce mouvement en le vidant de son contenu socio-économique mais en récupérant son contenu culturel implicite, un contenu donnant à la revendication autonomiste demie un véritable caractère d'authenticité. Dans ce contexte, l'attitude manifestée par la classe politique représentative de la majeure partie de la bourgeoisie, à l'égard du tahitien, prend tout son sens : elle s'inscrit essentiellement dans une stratégie de conquête de pouvoir. Ce faisant, je ne crois pas qu'elle ait l'intention de remettre en cause un seul instant, au moins en ce qui la concerne, le statut du français. Cette langue a constitué dans le passé un élément fondamental de sa promotion : à l'heure actuelle, avec le développement de l'enseignement secondaire, elle constitue plus que jamais l'outil intellectuel lui permettant de coloniser une administration pléthorique (bourgeoisie moyenne en plein essor) tout en lui permettant de conforter ses positions dans d'autres secteurs d'activité (haute bourgeoisie). Elle s'intègre donc toujours davantage à un système politique, administratif, économique, culturel, qui reproduit, moyennant quelques aménagements de pure forme, le système métropolitain. » (Ravault, 1982 : 103-104)

Ainsi donc, au regard de ce qui s'est passé en France métropolitaine où les langues régionales ont été défendues depuis longtemps par des spécialistes, des poètes, des monarchistes, des députés, en Polynésie française, contrairement à toute attente, c'est la bourgeoisie demie qui a impulsé le mouvement de reconnaissance de la langue tahitienne. En 1980, la langue tahitienne devint langue officielle du territoire de la Polynésie française, au même titre que la langue française $^{21}$. En 1984, la loi portant statut de la Polynésie française est adoptée à l'Assemblée nationale. L'article 90 de ce statut officialisait l'introduction des langues vernaculaires dans les écoles :

«La langue tahitienne est une matière enseignée dans le cadre normal des écoles maternelles et primaires. Cet enseignement est organisé comme matière facultative et à option dans le second degré. Sur décision de l'Assemblée territoriale, la langue tahitienne peut être remplacée dans certaines écoles maternelles et primaires, par l'une des autres langues polynésiennes. L'étude et la pédagogie de la langue et de la culture 
tahitiennes, seront, à cet effet, enseignées à l'École normale mixte de la Polynésie française. »

\section{En 1982, l'expression reo māòhi était déjà en} usage.

«On entend par reo māòhi, l'ensemble des langues polynésiennes du territoire de la Polynésie française. $»^{22}$

Tout est en place pour mener à bien une politique linguistique en faveur des langues polynésiennes. Et pourtant... !

\section{Langue populaire ou langue académique ?}

À l'évidence, la langue française garde encore son aura de langue de prestige, de langue de réussite sociale et professionnelle. Dans le milieu enseignant, cela tient à ce que l'on pourrait appeler un « conflit de loyauté », selon lequel l'enseignant ne s'autorise pas à comprendre le besoin de l'enfant à se construire à travers la langue locale, parce que lui-même tient sa réussite sociale et professionnelle de la langue française. Et pour les parents, l'école demeure un lieu d'apprentissage du français. Cette idée a longtemps marqué les consciences à tel point que fut notée, au fil du temps, une déperdition de plus en plus grande de la langue tahitienne à Tahiti et plus précisément dans la zone urbaine. Cette déperdition a sans doute été à l'origine de la création de l'expression « langue académique ». Voici comment Vonnick Bodin, alors directrice du CRFLC $^{23}$, aborde la question :

"Veut-on faire une langue orale, avec un enseignement oral, ou va-t-on l'écrire ? Et écrire quel tahitien, le langage populaire, ou le langage noble ?[...]. Malgré les heures de laboratoire... la finalité de l'enseignement de la langue n'est pas de parler couramment le tahitien, mais de bien l'entendre. Car ce qui s'entend bien se prononce bien. $»^{24}$

Ce discours sur le langage populaire ou noble est source de préoccupations chez les enseignants, qui sont encore d'actualité vingt ans après. S'agissant des radios et des télévisions, elles se sentent elles aussi préoccupées par la déperdition qui touche de plus en plus la langue tahitienne, notamment chez des jeunes, mais elles sont en même temps confrontées à l'audimat pour lequel une émission en tahitien, en particulier à la télévision, perd une part non négligeable d'audience comparativement à une émission en français. Le discours de Vonnick Bodin révèle un aspect réducteur de l'enseignement des langues qui peut prêter à sourire : si la finalité de cet enseignement n'est pas de parler couramment, abstraction faite de l'écoute énoncée plus haut, on est en droit d'être perplexe.

Dans les écoles, l'enseignement a-t-il été trop académique ? Le contenu des différents fascicules destinés aux élèves du primaire, ne semble pas l'affirmer. En revanche, l'idée d'une langue académique, d'une langue noble, a pénétré les esprits comme le montre ce passage de Bruno Saura, à propos d'un débat qui s'est tenu au Musée de Tahiti et des îles, le 19 octobre 1988 :

« Le thème de cette rencontre n'était autre que La langue, génie de la pensée (sous-entendu : La langue tahitienne, génie de la pensée tahitienne). De nombreux intervenants avaient alors pris prétexte de cet intitulé qui semblait fait pour eux, pour proclamer la richesse unique, incommensurable et incommunicable de la langue $m \bar{a} o ̀ h i$, dont la saveur propre ne pourrait qu'être dénaturée par l'exercice de la traduction, censée faire disparaître le génie de la langue. » $\left(1989\right.$ : 2) ${ }^{25}$

Cela nous donne une idée de l'ambiance dans laquelle pouvaient évoluer les enseignants. En réalité, elle les mettait dans une position inconfortable ; le génie de la langue māòhi (ici la langue tahitienne) ne permettrait jamais un enseignement tenu sur un discours peu élaboré aussi bien de la part du maître que celui de l'élève. À ce propos, une amie me confia qu'elle s'était détournée du tahitien (le restant de sa scolarité) à cause d'une parole blessante de son professeur pour une erreur minime qu'elle avait commise. L'exigence étant assurément très élevée, elle était source d'anxiété et obstacle à l'apprentissage.

Cette exigence a été confondue avec l'emploi d'une « langue académique ». Cette expression a été maintes fois entendue, souvent comme alibi, dès lors que l'accès à la compréhension faisait défaut. Or, à y regarder de plus près, il semble bien que la cause en est une grande déperdition de la langue constatée notamment au niveau des jeunes, mais pas uniquement. L'exemple le plus typique est le livre écrit pas Patrick Amaru ${ }^{26}$, que de nombreuses personnes ont beaucoup de difficulté à comprendre, et parmi elles des professeurs de tahitien du second degré. Et pourtant, dit l'auteur de ce livre, il n'a fait qu'utiliser des

22. Décision du Conseil de gouvernement 1021-SE du 20 octobre 1982.

23. Centre de formation et de recherche sur les langues et civilisations océaniennes.

24. La Dépêche de Tahiti (14. 02. 1984 : 10), cité par Bruno Saura (op. cit. : 122).

25. Duro Raapoto, co-organisateur de ce débat, y est nommément critiqué.

26. Te Oho na te tau 'Auhunera'a (« Les prémices du temps de l'abondance »), ce livre a reçu le prix du Président 2000. 
termes et des expressions qu'il avait entendus dans son enfance. Qu'il y ait une langue académique ou non, la principale préoccupation n'est pas là, elle est surtout dans la conciliation ou réconciliation autant des élèves que des professeurs avec la langue tahitienne, avec pour celle-ci un statut équivalent à celui du français; sans oublier pour autant la nécessaire et importante adaptation de la langue au public qu'elle veut toucher pour éviter les nombreux rejets dont elle fut victime.

\section{Une carence de plus en plus généralisée}

La question de la compétence des enseignants en langues polynésiennes a été une des raisons de l'abandon de la première expérience officielle de l'enseignement du tahitien dans le premier degré entamée depuis la rentrée scolaire 1976-1977. Voici ce que l'on peut lire dans la Dépêche du 16 mars 1977 :

« Or, c'est là qu'apparaît le handicap. Il ne s'agit pas seulement de "fabriquer" des instituteurs ayant un sens aiguë de la pédagogie, capables d'intéresser et de captiver une classe de vingt ou trente élèves de neuf et dix ans (l'expérience a lieu dans les classes de cours élémentaire deuxième année), encore faut-il que ces maîtres, tout volontaires qu'ils soient, possèdent un tahitien correct. Or, ce n'est pas le cas, c'est ce qu'ont pu découvrir, parfois avec beaucoup d'étonnement, les responsables de l'enseignement. Dans de telles conditions il apparaît impossible de généraliser l'enseignement du tahitien dans le primaire. Il faudra auparavant l'inculquer aux maîtres de l'École normale ».

Outre le problème de la politique linguistique, se pose celui du matériel didactique et des méthodes d'enseignement des langues polynésiennes, encore très insuffisants, et longtemps réclamés par les enseignants du premier degré. C'est à cette tâche que le ministre Nicolas Sanquer a voulu s'atteler en nommant au CTRDP deux personnes pour créer des outils pour la maternelle. Une équipe de bénévoles (composés de membres de l'Académie tahitienne, d'enseignantes spécialisées en maternelle) fut constituée autour d'elles. Elle reçut les félicitations de leur hiérarchie lorsque sortirent les premiers outils didactiques pour la section des grands. Il en fut de même pour celle des petits. Malheureusement, pour la section des moyens, le projet fut arrêté par une commission au motif que le contenu semblait inaccessible aux enfants. Il est vrai qu'ici le projet avait un contenu différent des deux autres (qui traitait de la réalité quotidienne), puisqu'il s'agissait d'aborder le merveilleux, l'onirique et de donner libre court à l'imagination de l'enfant au travers de contes et de légendes. La commission, en prenant cette décision, n'a jamais présenté d'arguments solides. Une nouvelle direction a donc été définie et une nouvelle commission, nommée «commission d'experts », mise en place pour orienter ce nouveau travail. Dans cette commission, plus de la moitié ne parle ni ne comprend le tahitien.

Ce récit n'est malheureusement pas une caricature. Le 28 novembre 2000 fut organisée la première « Journée des langues polynésiennes ». Cette journée décrétée par le gouvernement sera reconduite tous les ans. Si une avancée perceptible a pu être notée dans le domaine de la déclamation, dans sa vulgarisation, c'est qu'un travail immense a été mené en amont pour convaincre les anciens de laisser à la population la possibilité de s'approprier certaines connaissances. Sous l'égide du ministère de la Culture et avec la participation du ministère de l'Éducation, les deux premières années ont été indéniablement des réussites. On y a vu un engouement de plus en plus manifeste des établissements scolaires. La troisième année fut plus mitigée, le ministère de l'Éducation étant resté absent, sans aucune directive de sa part... De leur propre initiative, les établissements se sont organisés pour la circonstance.

\section{Faut-il enseigner les langues polynésiennes ?}

« À quoi sert-il d'enseigner les langues polynésiennes ? Le tahitien ça ne sert à rien, ce n'est pas ça qui va te permettre d'avoir un travail! »

sont les critiques le plus souvent entendues. Est-il exact que les langues polynésiennes ne servent à rien? ou pour dire les choses autrement : les langues polynésiennes ont-elles une utilité à l'école ? Comparativement à la France métropolitaine où, dans la vie quotidienne et familiale, on ne parle pratiquement qu'une seule langue, le français; en Polynésie française, l'enfant a la chance d'être au contact de plusieurs langues: une ou plusieurs langues polynésiennes, le français, parfois une langue étrangère... Jean Duverger et Jean-Pierre Maillard pensent qu'une compétence linguistique peut-être développée chez un enfant à travers un bilinguisme scolaire bien mené. L'enfant tirera bénéfice, d'une part, pour les deux langues et pour les apprentissages postérieurs, d'autre part, dans ce qui est appelé une « conscience métalinguistique ».

«On désigne ainsi le fait que l'enfant, manipulant deux langues, tant pour communiquer que pour s'exprimer et apprendre, prend conscience, d'abord 
intuitivement sans doute, puis de plus en plus explicitement, des règles quelquefois différentes et quelquefois semblables. En utilisant deux langues, il se développe inévitablement des stratégies d'analyses comparatives, voire contrastives, et ces analyses sont finalement bénéfiques à une connaissance plus fine et plus approfondie des fonctionnements des deux langues en présence. » (Duverger et Maillard, 1996 : 20)

Le bilinguisme apporte un autre atout « surtout s'il est précoce, il élargit considérablement les spectres actifs de l'ouie et de la phonation » (Duverger et Maillard, 1996 : 22). L'enfant, être en pleine croissance, dont les structures ne sont pas encore établies définitivement, voit donc s'offrir une multitude de possibilités d'acquisition linguistique. Dans son livre Entendre. Vivre par l'oreille de la conception à la vieillesse, Claude Rakowska-Jaillard tire les conclusions suivantes :

«- les bilingues sont plus capables de pensée abstraite que les monolingues, cela se constate dans leur façon d'appréhender les différentes disciplines scolaires, et notamment les mathématiques. On peut l'expliquer par le fait qu'ayant appris deux langues, ils ont deux symboles pour chaque objet et que, par conséquent, ils évaluent mieux la valeur des symboles; - les bilingues ont plus de flexibilité de pensée que les unilingues, grâce au fait qu'ils sont habitués à passer constamment d'un système de référence à un autre. Ils sont souvent avantagés par les connaissances qui leur viennent de deux cultures différentes. » (1982: 84)

Dans son étude sur les chants polyphoniques polynésiens, Raymond. Mesplé met en avant le fait que la musique en Polynésie est « relation au corps, comme le sont la langue, la danse et la sexualité » (1995 : 17). Plus loin, il ajoute :

« Les mélodies, rythmes et harmonies sont apprises par l'oral, et se transmettent avec des procédés de l'oralité également. L'imprégnation commence très jeune : il n'est pas rare de voir dans un groupe himene tārava [chant traditionnel] de jeunes mamans chanter avec leur bébé dans les bras. » (Mesplé, 1995 : 136)

Il faut donc profiter du caractère mélomane du Polynésien et tenir compte de cet aspect dans l'enseignement/apprentissage de la langue pour rappeler qu'elle est avant tout orale. À cet égard, voici ce que dit Claude Rakowska-Jaillard :

«L'écoute d'autres langues, surtout sous forme de chansons, n'aboutit certes pas à la connaissance de cette langue, mais marque les neurones de leurs modulations et de leurs rythmes, et plus tard en facilitera l'acquisition. Par ailleurs, un tel conditionnement laisse chez beaucoup d'individus une empreinte affective. » $(1982: 68)$
Selon ce même auteur, l'enfant peut apprendre deux et même trois langues avant l'âge scolaire (c'est-à-dire avant six ans).

« À cet âge, la classique crise d'opposition, la première, est généralement terminée, et la motivation existe : c'est le désir de communiquer. Il suffit que "l'autre" - la maitresse ou le petit camarade - soit sympathique, que s'établisse avec lui un contact affectif, pour que l'enfant soit motivé. Étant en pleine période d'imitation, il reproduira non seulement les sons, mais aussi le rythme, l'intonation, les attitudes corporelles. Ces dernières l'aideront considérablement à intégrer la langue. D'ailleurs, gestes et interjections font partie du langage. » $(1982: 81)$

\section{Quelle langue enseigner ?}

La question de l'accueil de l'enfant dans sa langue maternelle préconisé par le ministère de l'Éducation, hante de manière justifiée l'esprit de certains instituteurs, dès lors que sont accueillis en classe des enfants venus d'horizons divers. $\mathrm{Ce}$ serait une question quasi insoluble s'il fallait prendre cette directive au pied de la lettre. Dans son article Teaching of the Tahitian language in the schools in French Polynesia, Robert Levy opte pour un enseignement du tahitien dans toute la Polynésie française, puisque, selon lui, cette langue qui possède un statut de langue franche dans cette zone a été considérée comme la langue principale de communication en même temps qu'elle a développé une certaine conscience territoriale. Considérer cette proposition comme viable serait faire fi de l'existence des autres langues et donc de l'existence des autres communautés, d'autant qu'existent une Académie marquisienne et une forte activité associative pour la défense des langues paùmotu, mangareva... Dans les Objectifs et programmes du reo māòhi... paru en 1986, il est dit que :

« l'enfant de classe maternelle, et plus particulièrement de section des petits, sera [...] reçu dans sa langue maternelle. Lorsque le reo māòhi sera cette langue, il s'agira de la langue géographique de l'école (tahitien pour la Société, marquisien pour les Marquises...). Nous réalisons les dangers d'un tel choix pour les populations émigrées. Cependant, il est impossible de demander aux enseignants de recevoir chacun dans sa langue maternelle, dans les classes recevant des enfants d'origines diverses. Nous reconnaissons ces réalités culturelles et linguistiques, et nous optons pour un programme unique, applicable à toutes les classes maternelles du territoire, sans distinction aucune. »

Il va de soi que le programme malgré toutes les bonnes volontés de l'enseignant n'est pas applicable stricto sensu pour les raisons évoquées 
ci-dessus. Il peut y avoir consensus au niveau des directives, non au niveau des programmes. Ce consensus peut être trouvé lorsqu'il s'agit de considérer les langues polynésiennes en général. C'est-à-dire, montrer la proximité des langues entre elles afin de montrer à l'enfant l'appartenance de ces langues à une et même famille, et éveiller ainsi en lui une sensibilité plus grande et plus proche envers les autres peuples du Pacifique. En effet, le système éducatif français, par le contenu de ses programmes, a fini par modeler chez un grand nombre d'habitants une représentation erronée de l'aire géographique et culturelle de la Polynésie. Certaines personnes restent encore persuadées que des îles comme Wallis-etFutuna ou Samoa sont extérieures à cette aire ${ }^{27}$.

\section{La question de la graphie}

Les discussions sur la graphie du tahitien ont émaillé il y a quelques années encore des positions aussi tranchées et aussi dures que ne le fut la passion des personnages qui les anima. Pour exemple, la querelle qui opposa Maco Tevane ${ }^{28}$ et Jean-Marius Raapoto par articles interposés ${ }^{29}$ où celui-ci rejeta la graphie de l'Académie tahitienne qu'il estime être une contradiction à l'acte de lecture. En réponse, Maco Tevane rappela à son détracteur la présence du père de celui-ci en tant que membre de l'Académie qui adopta cette graphie $^{30}$ en 1976. Actuellement, dans les productions écrites, on rencontre différentes graphies. Néanmoins deux écoles de pensée se dégagent de manière distincte. Il s'agit de l'Académie tahitienne et de celle habituellement nommée « Turo ${ }^{31} »$.

Les textes qui utilisent la graphie de Turo sont ceux, surtout repérables, de l'Église évangélique. En revanche, ceux qui suivent la graphie de l'Académie tahitienne ne peuvent être définis de manière générale si ce n'est qu'en les désignant. En effet, dire que les institutions territoriales utilisent la graphie de l'Académie tahitienne, n'est vrai qu'en partie, puisque certaines utilisent celle de Turo, d'autres une combinaison des deux, ou d'autres encore écrivent sans accent. Visiblement, la graphie de l'Académie tahitienne ne fait pas l'unanimité. L'explication la plus plausible à cette situation serait due au fait que cette institution préconise, dans les textes scientifiques et didactiques, la notation de toutes les occlusives glottales et des macrons. À cela il faut ajouter les trémas et les traits d'union. Tout cela donne un texte lourd et rebutant à lire pour bon nombre de personnes. La question essentielle est de savoir si la notation de tous les signes diacritiques est nécessaire à la compréhension ? Cette question admet deux réponses : oui, lorsque le texte s'adresse à des personnes qui ne connaissent rien ou très peu le tahitien; non, lorsque le texte s'adresse à des gens connaissant le tahitien. Pour ceux qui sont très peu au fait de la langue, les signes diacritiques les aident à mieux « vocaliser » (ce qui, il faut le souligner, n'est pas encore un signe évident de compréhension), encore que, pour les Occidentaux, mais pas uniquement ${ }^{32}$, cela reste un exercice difficile, même avec un support auditif, en raison notamment de la prosodie et de la présence de nombreuses voyelles. On peut dès lors comprendre la position de l'Académie tahitienne, lorsqu'elle essaie de trouver des moyens pour faciliter l'apprentissage et la compréhension de la langue... Aussi peut-on se poser la question suivante: À quel public s'adresse-t-on lorsqu'on écrit en tahitien ? Selon toute vraisemblance, la graphie de l'Académie tahitienne s'adresse au néophyte, à celui non encore au fait de la langue. Ce qui pose une situation nouvelle pour des gens s'exprimant, lisant et écrivant facilement un tahitien dépourvu de signes diacritiques. Comment fontils? Suivent-ils ou non la graphie préconisée ? Dans la réalité, ils adoptent la graphie la plus simple pour eux et compréhensible en même temps par les autres. C'est, selon Jean-Marius Raapoto (linguiste et professeur de tahitien), le choix adopté par les missionnaires lors de la rédaction de La Bible. Il y avait chez eux le souci d'éviter, en même temps, toute confusion de sens et toute surcharge. On peut donc légitimement penser que la présence de signes diacritiques n'est pas indispensable à la compréhension. En effet, selon le témoignage d'une maîtresse ${ }^{33}$ d'école, des enfants de cours préparatoire et de cours élémentaire première année étaient capa-

27. On peut d'ailleurs noter à ce sujet que les médias utilisent fréquemment Polynésie en lieu et place de Polynésie française.

28. De son vrai nom Marc Maamaatuaiahutapu, il est directeur de l'Académie tahitienne.

29. Lire les Veà porotetani (9 novembre 1996 et 11 février 1997).

30. Cela n'a aucun sens. Une opinion reste personnelle, elle se dégage de toute appartenance parentale.

31. Il s'agit de Duro Raapoto. Il est linguiste et professeur de tahitien. Bien que la première école soit une institution officielle, la seconde n'en est pas moins considérée comme telle par des personnes qui se retrouvent en elle. Pour la commodité de notre discours, nous désignerons celle-ci par « Turo ».

32. En fait, il s'agit de problème de compétence et de pratique de la langue, puisque même chez les natifs du pays, certains éprouvent des difficultés de prononciation.

33. Elle a participé à une expérience d'enseignement en langue tahitienne pour des enfants en grande difficulté scolaire. 
bles de lire, sans trébucher, des textes en tahitien dépourvus de signes.

Contrairement à la graphie « dite officielle ${ }^{34}$ » qui éclate la structure du mot, celle de Turo garde celle-ci intacte. L'Académie tahitienne considère l'occlusive glottale comme une consonne. Elle lui attribue donc une place dans la structure du mot, par exemple: to' $i$ (herminette), alors que le même terme dans la graphie de Turo s'écrit comme celle-ci : toì ; l'occlusive glottale se place sur le $i$. Là n'est pas la seule particularité de cette écriture liée. En effet, Turo a constaté que, d'une manière générale, lorsque deux mêmes voyelles se suivent, la deuxième prend (inévitablement) un coup de glotte. Il n'y a donc pas nécessité de l'écrire. Ainsi, aura-t-on : $a a$ [a? a] pour racine, rii [rî i] pour petit... Lorsque l'on a affaire à des mots d'une certaine longueur, on peut avoir les graphies suivantes : ha'api'ira'a (enseignement) pour l'Académie tahitienne, et haapiiraa pour Turo. Pour peu qu'il y ait réduplication (partielle), on aura : ha'api'ipi'ira'a et haapiipiiraa. L'utilisation des signes diacritiques n'est pas normalisée. Le sera-t-elle un jour? Certaines personnes interrogées à ce sujet pensent que «non ». Les prises de position, non dénuées d'esprit partisan viennent nous rappeler ces difficultés. À l'heure actuelle, rien ne semble tendre vers la recherche d'un consensus. Bien au contraire, en se positionnant comme défenseur (à sa manière) de la langue māòhi, l'Église évangélique prend le choix d'officialiser par là même la graphie préconisée par Turo. Des tentatives ont pourtant eu lieu.

Mais au-delà de cette querelle graphique, il convient de rappeler la volonté de faciliter l'accès à la lecture et à l'écriture des langues polynésiennes du plus grand nombre. Or, la graphie de l'Académie tahitienne, pour reprendre l'expression de Jean-Marius. Raapoto, est une contradiction à l'acte de lecture. Loin de faciliter les choses, cette institution, selon certains propos, a décidé de mettre une glottale à la particule 'ua, sous prétexte qu'elle viendrait de kua, afin de remplacer le $k$ disparu, alors que cette particule se prononce $[u a]$. On a donc l'impression de voir s'exprimer ici une volonté délibérée de complica- tion, afin semble-t-il de rendre les choses plus claires, ou peut-être plus vraies, si ce n'est plus authentiques, comme si le passé était plus vrai que la vérité réelle vécue par les gens d'aujourd'hui. Complication dont les quelques érudits détiendraient les clés tant les méandres relèvent $\mathrm{du}$ parcours du combattant. Combien de fois n'ai-je vu une amie, pourtant bien au fait de la langue tahitienne, prendre son dictionnaire pour vérifier si l'utilisation des accents est bien justifiée! Avec la préoccupation permanente d'utiliser la "bonne" graphie, comment peut-on espérer inciter les gens à lire et à écrire en tahitien ? D'autant que l'acte de lire et l'acte d'écrire ne semblent pas être réellement une seconde nature chez le Polynésien.

La réponse à cette question réside dans la réduction au minimum des contraintes ${ }^{35}$. Or, l'idée même de proposer cet apprentissage à partir de l'écrit (en essayant notamment de rendre correcte la prononciation des mots) est une contradiction dans l'apprentissage d'une langue, qui plus est dans une société davantage marquée par l'oralité que par l'écrit. Il est fort probable que les personnes qui ont codifié la langue ont perdu de vue les aspects pratiques liés à la lecture et à l'écriture. Par ailleurs, le livre comme instrument d'apprentissage demeure fortement présent dans notre conscience. Or, il faut le reconnaître, tout apprentissage d'une langue nécessite un passage obligé par l'oral. L'Académie marquisienne a fait le choix d'une écriture liée, celle préconisée par Turo ${ }^{36}$. Cette décision n'a pas été sans créer une réaction du ministre de la Culture lui demandant de « revoir sa position $»^{37}$. Sur les ondes de Radio Marquises, les Marquisiens ont fait savoir leur total accord avec le choix opéré par l'Académie marquisienne. En revanche, il est contesté par les autorités religieuses catholiques.

\section{Conclusion}

Il est indéniable, comme nous venons de le voir, que les langues polynésiennes ont leur place dans l'enseignement, et que revient à l'école le rôle prépondérant. S'il est difficile de changer les

34. Cette nuance rappelle que le caractère officiel de l'institution ne présage en rien d'un usage effectif et total de ses choix et décisions, par la collectivité. Il me semble par ailleurs qu'aucun texte ne mentionne l'officialisation de la graphie de l'Académie tahitienne, si ce n'est une décision de 1982 émanant du service de l'Éducation qui oblige le CTRDP à se conformer aux normes linguistiques établies par l'Académie tahitienne. Cette même décision est en vigueur au concours de CAPES bivalent français/tahitien.

35. Ajoutons que cette mesure, si elle enlève certains obstacles, devrait être suivie de mesures d'accompagnement, notamment au plan auditif (cédéroms...).

36. L'idée maîtresse est la réduction au minimum des signes diacritiques. Signalons au passage que l'INALCo adopte le code graphique proposé par Duro Raapoto (cf. Mirose et Vernaudon, 1998).

37. On peut d'ailleurs se poser la question de la légitimité du ministre à remettre en cause une décision prise par une institution. 
habitudes langagières des parents, en revanche l'école (si les mesures sont mises en place) a la particularité et la capacité de se moduler et de s'adapter. Ce qui importe, c'est de parier sur l'avenir. Toute la question est de savoir si les décideurs sont convaincus du rôle important que peuvent tenir les langues polynésiennes, au-delà des discours politiquement corrects, maintes fois entendus. De nombreux pays occidentaux sont de plus en plus convaincus de la nécessité de garder et d'enseigner les langues minoritaires. Des projets de sauvegarde sont financés par des fondations ${ }^{38}$. Nous avons vu plus haut que des concours de littérature écrite sont organisés tous les ans, ainsi qu'un concours de déclamation (òrero), auxquels participent de nombreux établissements scolaires. L'évangile de Luc paru en film a été traduit en tahitien. De plus en plus d'adultes veulent apprendre le tahitien (mais aussi le marquisien, le paùmotu, le mangaré$\left.v{ }^{2}{ }^{39} . ..\right)$ et se trouvent parfois malheureusement confrontés au manque de professeurs... Le contexte est donc favorable pour lancer des initiatives afin de dynamiser cet enseignement. L'une d'entre elles est ce que Claude Hagège nomme : «le principe de Ronjat », principe qui admet : une personne, une langue (c'est-à-dire : un enseignant, une langue). Cette manière d'enseigner la langue est facilement applicable en primaire. Pour l'instant, le ministre de l'Éducation (de Polynésie française) s'en tient à l'idée de la polyvalence des enseignants ${ }^{40}$ et, à cet égard, il a supprimé la fonction de conseiller pédagogique en langues polynésiennes. Or, beaucoup d'enseignants, comme nous l'avons vu depuis les premières expériences, ne maîtrisent pas le tahitien. La formation à l'École normale n'a pas donné les résultats espérés. La situation s'enlise, pis ! elle se dégrade. Par ailleurs, la tentation devient trop forte d'utiliser les heures réservées à l'enseignement des langues polynésiennes pour combler les retards du programme scolaire. Avec l'application du principe de Ronjat, on élimine tout cela. L'enseignant qui aura cette charge sera choisi en fonction de sa compé- tence en langues. Il enseignera dans plusieurs classes à l'image des professeurs du second degré qui n'ont qu'une matière à enseigner. Ainsi, il aura une grande liberté d'action pour mettre en place un programme et une progression cohérents et suivis dans le temps, et si possible, adaptés à différents niveaux. L'alibi du retard dans le programme scolaire n'aura plus cours ici, puisque les heures prévues seront effectives.

Cette expérience est menée actuellement à l'école de Mangareva depuis quelques années ${ }^{41}$... Les résultats sont prometteurs et les parents se réjouissent des effets bénéfiques sur la scolarisation des enfants, de la complicité qui a pu naître entre l'enfant, l'école et les parents, mais aussi du changement radical de l'enseignante qui autrefois interdisait aux élèves de parler la langue mangarevienne ${ }^{42}$. Phénomène inattendu, le cahier qui suit l'enfant durant toute sa scolarité est devenu le symbole fort d'un lien intergénérationnel ${ }^{43}$. Le principe de Ronjat ou loi Grammont-Ronjat, a montré d'excellents résultats ailleurs. Voici ce qu'en dit Claude Hagège :

«L'application du principe de Ronjat donne des résultats satisfaisants, si l'on en juge par les cas les plus connus, et d'abord par le premier d'entre eux, celui de l'enfant Louis Ronjat, qui acquit assez vite une connaissance remarquable du français et de l'allemand [...]. Cela ne signifie pas, évidemment, que cette application soit le facteur décisif, ni que l'alternance des langues chez les parents ait nécessairement des effets négatifs sur l'apprentissage en provoquant la confusion. Cela signifie simplement que les chances de succès mesurées d'après les observations de spécialistes, sont plus fortes lorsque l'on respecte le principe de Ronjat que dans le cas contraire. » (Hagège, 1996b : 43)

Il est assez intéressant de noter ici la pertinence de réflexions d'enseignants qui vont dans le même sens que ce qui est dit plus haut.

«Même [dans] la structure actuelle, c'est mal fait. Moi je pense, qu'on devrait avoir une personne spécialisée en reo màòhi, qui passerait dans les classes et ne parlerait qu'en reo māòhi, parce que [s'agissant de]

38. Actuellement la fondation Volkswagen finance une vingtaine de projets de sauvegarde de langues à travers le monde, dont celui sur la langue et la culture marquisiennes.

39. Le paùmotu et le mangarévien sont respectivement les langues des archipels Tuamotu et Gambier.

40. Cette décision est comprise comme une mesure permettant d'éviter tout précédent qui risquerait de provoquer une demande trop importante d'enseignants spécialisés, n'enseignant qu'une discipline. Dans le cas qui nous occupe ici, il faut savoir que la langue n'est pas une discipline comme les mathématiques, l'histoire... elle est le véhicule, l'outil... avec lequel on enseigne une discipline. Il en est ainsi du français, de l'anglais comme des langues polynésiennes... Cette décision pour autant qu'elle doive sembler légitime aux yeux des autorités, n'en est pas moins caractéristique du statut que ces mêmes autorités accordent aux langues polynésiennes.

41. Cette affirmation vient contredire les propos tenus plus haut. Il s'avère que des expériences de ce genre ont déjà eu lieu de manière éparse et épistolaire. Les raisons de leur mise en place peuvent être multiples, parfois ne relevant pas spécifiquement de la pédagogie, mais cela ne présageant en rien de la qualité du travail.

42. Sans doute perçu comme une sorte de rachat, d'image rétablie et de conscience collective légitimée.

43. C'est ainsi que certains parents ont pu se réapproprier leur histoire et leur culture. 
nous, les enfants savent très bien qu'on [ne] va parler [qu'] en français. Ils ne vont pas chercher à comprendre. De toute façon, ils vont se dire : "Bon, Monsieur va expliquer [en français]..." Il faut qu'il y ait une autre personne, et là, ils seront forcés de réfléchir, de s'intégrer... Pour eux, c'est pas sérieux, ils rigolent. Ils se disent : "C'est bizarre, pourquoi il nous parle en tahitien, alors qu'il sait parler en français." Allez imaginer ce qui se passe dans leur tête. C'est pour ça, moi, il faudrait une personne spécialisée si on veut vraiment enseigner le reo mäòhi dans les écoles. Il faut une personne spécialiste qui ne parle que cette langue. »

Il faudrait que les décideurs sortent de leur frilosité et généralisent l'expérience de Mangareva. Les conditions sont réunies : une compétence décisionnaire territoriale et des enseignants volontaires...

Le sentiment de déperdition des langues polynésiennes, relevé plus haut, notamment du tahitien, a incité certains parents de la commune de Paopao à Moorea à se constituer en association et à mettre en place une école comparable au Kohanga reo (nid de la langue) de NouvelleZélande, au Punana leo (nid de la langue) de Hawaii, aux écoles Diwan de Bretagne ou aux Ikastola du pays basque... Le fonctionnement de cette école appelée Puna reo repose sur la bonne volonté des gens et a ouvert ses portes au mois d'octobre 2003. Elle fonctionne tous les samedis durant toute la journée.

« Durant les vacances d'octobre, les animateurs de l'école Puna reo se rendront en Nouvelle-Zélande retrouver les enseignants d'un établissement kohanga reo qui pratiquent le bilinguisme en immersion totale, de la maternelle à l'université. $»^{44}$

Paradoxe! Les Kohanga reo, ainsi d'ailleurs que les Punana leo, se sont inspirés d'une expérience qui a mobilisé les habitants de la commune de Paopao vers les années 1976-1977. Des Maoris et des Hawaiiens se sont déplacés pour cette occasion.

La presse s'était fait l'écho de l'échec de l'intégration des écoles Diwan dans l'enseignement public. Intégration qui aurait permis de supporter une grande partie des charges financières. Pour débloquer la situation, le ministre Luc Ferry a demandé aux écoles Diwan un effort d'adaptation en aménageant le quart des cours en français. La mise en place des écoles en immersion en Polynésie française n'est pas exempte de ce genre de difficultés. Un échec risquerait de porter préjudice à l'image de ces écoles et à leur continuité, et donnerait ainsi raison aux sceptiques. En essayant de tirer profit de la proposition faite par le ministre de l'Éducation aux écoles Diwan, l'intégration de l'école Puna reo dans l'enseignement public est tout à fait opportune.

\section{BIBLIOGRAPHIE}

BARÉ Jean-François, 1985. Le malentendu pacifique, Paris, éd. Hachette.

BULLETIN PÉDAGOGIQUE ET ADMINISTRATIF DES INSTITUTEURS ET INSTITUTRICES DES ÉTABLISSEMENTS FRANÇAIS D'OCÉANIE 1, 1939 (avril), Papeete.

— 2, 1939 (juillet).

BULLETIN OFFICIEL DES ÉTABLISSEMENTS FRANÇAIS D'OCÉANIE 10, 1857 (octobre).

- 11, 1857 (novembre).

Calvet Louis-Jean, 1974 (rééd. 1979). Linguistique et colonialisme, petit traité de glottophagie, Paris, Payot, Petite bibliothèque.

Duverger Jean et Jean-Pierre MaIllard (en collaboration avec), 1996. L'enseignement bilingue aujourd'hui, Paris, Albin Michel, bib. Richaudeau.

HAGÈGE Claude, 1996a. Le français, histoire d'un combat, Paris, éd. Michel Hagège.

—, 1996b. L'enfant aux deux langues, Paris, éd. Odile Jacob.

LEVy Robert, 1970. Teaching of the Tahitian language in the schools in French Polynesia, in Journal de la Société des Océanistes 26, XXXVI, pp. 79-83.

Male Émile, 1974. L'enseignement protestant en Polynésie française, mémoire de licencié en Théologie protestante, université de Strasbourg, IER de Théologie.

Mesple Raymond, 1995. Hymnologie protestante et acculturation musicale à Tahiti et en Imerina (Madagascar), doctorat en ethnologie, université de la Réunion, faculté des Lettres et Sciences humaines, centre d'Anthropologie généralisée.

Mirose Paia et Jacques Vernaudon, 1998. Tahitien, méthode de langue, Paris, InALCo/Centre Georges Pompidou.

Moyse-Faurie Claire, 2000, Les langues minoritaires et politiques linguistiques : le cas des langues océaniennes, éd. Peeters, Mémoires de la Société de linguistique de Paris, pp. 79-104.

O'ReILly Patrick, 1982. Tahiti, la vie de chaque jour, Paris, Nouvelles éditions latines.

Platten, 1951. Pédagogie, Bulletin de la Société d'études océaniennes (BSEO) 97, pp. 293-301.

Poignant Bernard, 1998. Langues et cultures régionales, Paris, La Documentation française, collection des rapports officiels 
_, 2000. Langues de France : osez l'Europe, Montpellier Indigène éditions.

RAKOWSKA-JAILlaRD Claude, 1982. Entendre, Vivre par l'oreille de la conception à la vieillesse, Paris, Éditions universitaires.

Ravault François, 1982. Le français dans une société pluri-culturelle, l'exemple de la Polynésie, Anthropologie et Sociétés 6, 2, pp. 89-105.

ReY-Lescure Philippe, 1951. La langue maternelle, Bulletin de la Société d'études océaniennes (BSEO) 97, pp. 304-305.
SAURA Bruno, 1986. L'identité polynésienne : facteur de revendication et discours identitaire à Tahiti, DEA d'études politiques, uinversité de Droit, d'Économie et des Sciences d'Aix-Marseille III.

—, 1989. Théologie de la libération et théorie de la culture chez Duro Raapoto, Bulletin de la Société d'études océaniennes (BSEO) 246, pp. 1-15. 
